Referencia para citar este artículo: Aguirre, F., Cruz, M. A., Banda, M. I. (2018). La dimensión sociocultural de la globalización en relatos sobre una experiencia de movilidad internacional universitaria. Revista Latinoamericana de Ciencias Sociales, Niñez y Juventud, 16(2), 881-895. doi:https://doi.org/10.11600/1692715x.16216

\title{
La dimensión sociocultural de la globalización en relatos sobre una experiencia de movilidad internacional universitaria*
}

\author{
FÉLIX AGUIRRE** \\ Profesor Universidad de Valparaíso, Chile. \\ MaRÍA ANGÉLICA CRUZ ${ }^{* * *}$ \\ Profesora Universidad de Valparaíso, Chile. \\ MARÍA IGNACIA BANDA **** \\ Asistente de investigación Universidad de Valparaíso, Chile.
}

\section{Artículo recibido en octubre 24 de 2017; artículo aceptado en enero 25 de 2018 (Eds.)}

- Resumen (analítico): este trabajo analiza la experiencia de un grupo de jóvenes estudiantes daneses en la Universidad de Valparaíso, en el marco de un programa de movilidad internacional. El uso de relatos de vida nos permite describir no sólo las formas en que esta experiencia transforma sus identidades y sus trayectorias académicas, profesionales e ideológicas, sino también sus imaginarios sobre los lugares de origen y destino. Los hallazgos contribuyen a la discusión sobre la dimensión sociocultural y política de la globalización, en particular la articulación entre los valores de una ciudadanía global y las diferentes formas de comprender y vivir la alteridad.

Palabras clave: globalización, universidad, movilidad estudiantil internacional, valores sociales, ciudadanía, método biográfico, alteridad (Tesauro de Ciencias Sociales de la Unesco).

\section{The sociocultural dimension of globalization in narratives about experiences of international university exchanges}

- Abstract (analytical): This paper analyzes the experiences of a group of young students from Denmark who studied at the University of Valparaiso as part of an international university exchange program. The life story methodological technique allows us to describe not just the way in which the experience of participating in an exchange program transforms students' identities and academic, professional and ideological trajectories, but also how it affects their perceptions and concepts of

Este artículo de investigación científica y tecnológica presenta los resultados de la investigación «Voces sobre la globalización. Las dimensiones de la movilidad internacional y el fenómeno sociocultural de la internacionalización en una muestra de estudiantes extranjeros que eligieron la Universidad de Valparaíso como destino para complementar su formación de pregrado», financiada por la Dirección de Investigación de la Universidad de Valparaíso (Chile), en su convocatoria Diuv-REG Na51/2011, investigación iniciada en marzo de 2013 y finalizada en marzo de 2015. Gran área de conocimiento: Ciencias Sociales; Área de Conocimiento: Sociología; Subárea: Temas Sociales.

** Sociólogo de la Universidad Complutense de Madrid, España. Doctor en Ciencias Políticas y Sociología por la Universidad Complutense de Madrid, España. Profesor Titular del Instituto de Sociología de la Universidad de Valparaíso. Orcid: 0000-0002-9527-5757. Índice H5: 2.Correo electrónico: felix.aguirre@uv.cl

*** Socióloga de la Universidad de Chile. Doctora en Sociología por la Universidad Complutense de Madrid. Profesora Titular del Instituto de Sociología de la Universidad de Valparaíso. Orcid: 0000-0002-6958-859X. Índice H5: 2. Correo electrónico: mariaagelica.cruz@uv.cl

**** Socióloga de la Universidad de Valparaíso. Magíster en Género e Investigación, London School of Economics and Political Science. Orcid: 00000003-1281-1743. Correo electrónico: igbandac@gmail.com 
their places of origin and destination. The findings from this study contribute to the debate about the sociocultural and political dimensions of globalization, particularly on links between the values of global citizenship and the different ways of understanding and living alterity.

Key words: Globalization, university, international student mobility, social values, citizenship, biographical method, alterity (Unesco Social Sciences Thesaurus).

\section{A dimensão sociocultural da globalização em relatos sobre uma experiência de mobilidade internacional}

- Resumo (analítico): o trabalho analisa a experiência de um grupo de jovens estudantes dinamar-queses na Universidade de Valparaíso, no contexto de um programa de mobilidade internacional. O uso dos relatos de vida nos permite descrever não somente as formas em que esta experiência transforma suas identidades e trajetórias acadêmicas, profissionais e ideológicas, mas também os seus imaginários sobre os lugares de origem e destino. Os resultados contribuem à discussão sobre a dimensão sociocultural e política da globaliza-ção, em particular à articulação entre os valores de uma cidadania global e as diferentes formas de compreender e vivenciar a alteridade.

Palavras-chave: globalização, universidade, mobilidade internacional dos estudiantes, valores sociais, cidadania, método biográfico, alteridade (Thesaurus de Ciências Sociais da Unesco).

-1. Introducción. La movilidad estudiantil y la internacionalización de la universidad más allá de las cifras. -2. Los alcances teóricos y el problema de investigación. -3. Nota metodológica. -4. Resultados. $\mathbf{- 5}$. Reflexiones finales. -Lista de referencias.

\section{Introducción. La movilidad estudiantil y la internacionalización de la universidad más allá de las cifras}

Durante los últimos treinta años el proceso de incorporar una dimensión internacional, intercultural o global a los objetivos, las funciones y la oferta de la educación universitaria se ha acelerado notablemente, en buena medida, debido a un crecimiento sostenido de la cantidad de estudiantes extranjeros que anuncia la intensificación de la competencia para atraerlos ante la eventual emergencia de otros países llamados a incorporarse a la lista de receptores de los más de cuatro millones y medio de estudiantes internacionales que tendremos hacia el 2020 (Ritzen, 2006). Aunque la mayoría de las universidades se han visto afectadas, forzadas o motivadas a gestionar este proceso, las políticas de internacionalización han sido más bien dispersas e inorgánicas (Unesco, 2009). Ello se refleja en que prácticamente no existen instrumentos de evaluación que permitan comprender este fenómeno, de forma que si sabemos poco acerca de cómo estas experiencias afectan a los resultados del proceso educativo formal, sabemos mucho menos sobre cómo interviene en la transformación de valores, normas y comportamientos (Tarabini \& Bonal, 2011).

En el flujo mundial de exportación y recepción de estudiantes de intercambio los países en vías de desarrollo han desempeñado, por lo general, un rol pasivo en un mercado mundial que adolece de notorias asimetrías, en tanto es nacionalmente segregado de acuerdo a ingresos e internacionalmente desproporcionado según la posición del país (Lörz, Netz \& Quast, 2016). Por ejemplo, mientras que ninguno de los países de América Latina figura actualmente en la lista de las veintitrés naciones que atraen mayor número de estudiantes internacionales, en Brasil, México y Argentina la constante migratoria es muy inferior a la que registran los principales países receptores desarrollados: apenas el uno por ciento del estudiantado matriculado en la educación terciaria es extranjero y una cifra similar emigra para cursar estudios superiores en otros países, una asimetría que pareciera estar asociada a factores como la escasez de universidades locales con una calidad suficiente para satisfacer la 
demanda de los mercados internaciones, con la ausencia de incentivos a esta movilidad en el marco de políticas públicas de educación terciaria, o con el hecho de que la misma ha sido históricamente financiada privadamente por las élites nacionales (Rodríguez, 2005).

Chile no ha sido la excepción. Lo que comenzó siendo una moda espoleada por el incentivo que suponían los programas de movilidad ensayados con éxito desde mediados de la década de 1990 en los países europeos, también en el país hemos asistido a la rápida conformación de lo que es ya un fenómeno transcultural, con todos los ingredientes necesarios para poder ser identificado con el apelativo de global. Dicho con otras palabras, la internacionalización a que induce la movilidad y la migración estudiantil no es sino la respuesta al escenario global que enfrentan las universidades chilenas (Knight, 1999).

Aunque los motivos por los cuáles se busca fortalecer este proceso obedecen a razones tanto académicas como económicas, cada año más estudiantes internacionales eligen Chile como destino. Solamente en las universidades de la región de Valparaíso el número de estudiantes extranjeros se triplicó entre el año 2003 y el 2013 (Servicio de Información de la Educación Superior, 2013). Las movilizaciones estudiantiles - y las consecuentes paralizaciones - que se agudizaron en todo el país a partir del año 2011 no fueron impedimento para que 166 jóvenes llegaran el año 2013 a la Universidad de Valparaíso. Es más, todo indica que muchos de los estudiantes extranjeros se sumaron a tales movilizaciones activamente y que ello supuso una vivencia relevante en su experiencia de movilidad.

En este contexto, realizamos una pesquisa con el objetivo de sumar evidencia al debate sobre la conformación de una idea de ciudadanía, particularmente entre los y las jóvenes, que pudiera ser comprendida más allá de las fronteras del Estado-Nación. Se trata de una tendencia que aparece en el contexto de una progresiva cristalización de valores globales, a la vez que la creciente problematización de sus implicancias ideológicas y políticas. Por ello, partiendo de la premisa de que la movilidad ha de entenderse también en términos de una nueva concepción axiológica (Muñoz, 2004; Tarrant, 2011), la presente investigación se inserta en tal discusión, indagando en los sentidos actuales de la movilidad estudiantil para luego preguntarse por los alcances de esta tendencia en el marco de transformaciones culturales de largo alcance. A partir de relatos de vida realizados con estudiantes daneses que cursaron durante el año 2011 un semestre en la Universidad de Valparaíso, en el marco de un programa de intercambio con la Universidad de Aalborg, exploramos el impacto de esta experiencia en las trayectorias de vida juveniles de procedencia nórdica y cultura socialdemócrata, para volver sobre la idea de ciudadanía global, las tensiones y los desafíos que ello supone.

En lo que sigue, tras presentar los principales referentes teóricos y metodológicos, exponemos una selección de los resultados. Para ello hemos ordenado las experiencias de los y las estudiantes que participaron en nuestro estudio en dos ejes. El primero, presenta la reconstrucción de la experiencia de intercambio en un continuo que va desde una dirección unívoca (privilegiando sobre todo un aspecto del viaje) a una múltiple, donde se mezclan aprendizajes personales, académicos y políticos. El segundo eje corresponde a las cuatro dimensiones de la ciudadanía global que propone Tarrant (2011), que van desde un grado básico de compromiso con el nuevo entorno al que se llega a estudiar, hasta un alto nivel de involucramiento social. A partir de tal descripción, nos preguntamos por cómo estos jóvenes estudiantes abordan su relación con los no europeos, con «el otro» —representado por quienes habitan en Valparaíso, Chile o Latinoamérica, según los lugares que hayan podido visitarusando para ello ciertas metáforas de la antropología. Esto nos permite identificar algunos desafíos que enfrentan los procesos de internacionalización universitaria en Latinoamérica, a diferencia de sus símiles europeos o norteamericanos. Lo anterior interpela a ambas partes en la apuesta por una construcción crítica y decolonial de la alteridad, una discusión que, a nuestro juicio, debiera atravesar cualquier propuesta de ciudadanía global. 


\section{Los alcances teóricos y el problema de investigación}

La globalización es un proceso complejo del que generalmente sólo se destacan sus consecuencias económicas. Sin embargo, tomada como la dimensión cultural del orden moderno, es posible plantear una reflexión de mayor rendimiento explicativo. Distintos autores han desarrollado enfoques para tratar de caracterizar la sociedad contemporánea como una sociedad mundial (Castel, 2006; Habermas, 1986; Luhmann, 1990;) en la que es posible comprender los procesos en curso mediante una concepción desterritorializada de lo social, y donde los individuos y agentes (individuales o colectivos) forman parte de un sistema único de comunicación. La sociedad mundial de la globalización supone también transformaciones en la subjetividad y en los procesos de construcción de identidad como marco de sentido para la acción (Melucci, 2010), lo que implica tensiones entre lo propio o lo particular y lo global, puesto que se tienden a desarrollar conjuntamente procesos de homogenización y diferenciación identitaria.

La dimensión cultural de la globalización ha sido, quizá, el proceso de mayor resistencia, en tanto viene a tensionar las tradicionales concepciones densas de la cultura. La extensión de estilos de vida crecientemente homogéneos «obliga» a pensar lo propio, ya no en referencia a sí mismo sino en tensión permanente con modelos que se imponen a través de la masificación del consumo y por el acceso universal a los mensajes y contenidos a través de los medios de comunicación, lo que homogeniza tanto los estilos de vida como las maneras de pensar, sentir y actuar (Giddens, 1994).

Los programas de estudio en el extranjero ofrecen una experiencia vital que supera con creces el impacto de la instrucción tradicional, y nos induce a plantear la necesidad de comprender este proceso y sus alcances (Van Mol, 2017). El supuesto más recurrente es que estudiar en el extranjero promueve una cosmovisión y una conciencia de los problemas mundiales (Dolby, 2007). Sin embargo, aún contamos con poca evidencia que nos provea de los marcos conceptuales necesarios para explicar cómo sucederían tales procesos. El estudio ya clásico de Lucas Luchilo (2006) identifica tres formas habituales de entender la movilidad estudiantil; i) como una lógica de cooperación entre estados; ii) como una herramienta para atraer personal académico y científico calificado; y iii), como una fuente capaz de proveer ingresos extraordinarios. Sin embargo, el autor deja claro que sus efectos más bien heterogéneos nos obligan a explorar el fenómeno de la movilidad en un contexto de significación cultural de mayor calado.

Algunas investigaciones recientes centran su atención en los alcances de la experiencia de movilidad en la conformación de identidades profesionales (Bell, Moorhead, \& Boetto, 2015), en la conformación del pensamiento crítico (Nguyen, 2012) o en el desarrollo de competencias globales (Hunter, White, \& Godbey 2006); mientras que otros estudios analizan el potencial de este tipo de movilidad en la confrontación de barreras y prejuicios etnocéntricos, al verse desafiados los estudiantes por el paso de un entorno familiar a uno imprevisto, un proceso potencialmente conducente a una conciencia distinta y, por tanto, de uno/a mismo/a (Bell et al., 2015). De la misma forma, se discute sobre el potencial de la movilidad estudiantil en el desarrollo de una conciencia social global en los y las jóvenes, muchas veces dependiendo de la duración del programa (Pugh, 2013) o de su coherencia en el marco de una formación profesional en el área social (Bell et al., 2015), donde la confrontación de los estudiantes con realidades fuera de su alcance es vista como una potencial ventaja. Lo cierto es que las tensiones entre este potencial y sus resultados no son del todo pronosticables (Mendoza, Staniscia, \& Ortiz, 2016). Si bien la literatura insiste en las ventajas del encuentro entre culturas distintas (Bowman \& Jennings, 2005; Ungar, 2016), la discusión también hace notar el carácter profundamente complejo y multidimensional del mismo (Doerr, 2016).

El trabajo de Michel Andrew Tarrant (2011) centrado en el análisis de la emergencia de valores medioambientales en estudiantes internacionales, retoma el modelo de la teoría Value-Belief-Norm (VBN). De acuerdo a este modelo, la emergencia contemporánea de valores transculturales, como el altruismo, parece potenciada por la creciente socialización de los y las jóvenes en un conjunto de creencias sobre las consecuencias que tienen los actos propios en otros sujetos, y por la obligación de 
actuar para prevenir esas potenciales consecuencias (Stern, 2000). El modelo VBN apunta a la idea de que la experiencia vital de los estudiantes internacionales puede alimentar una ciudadanía universal que se identifica con un concepto de justicia, útil para distinguir entre una comunidad de ciudadanos y otra de meros seres humanos. En consecuencia, un «buen ciudadano» es aquel que acepta una obligación política para actuar de manera justa e imparcial, en contraste con el «buen samaritano» que puede actuar sin un claro sentido del deber. Una distinción que, en el caso del estudio mencionado, se ilustra con el ejemplo del cambio climático, pero que resulta extensible a un escenario global, donde la obligación del ciudadano no solamente es cívica, sino también no-recíproca; es decir, la obligación beneficia a personas con quienes no se tiene una relación inmediata. En definitiva, la emergencia de un «ciudadano-tipo» se caracterizaría por ser: i) alguien que actúa responsablemente en su comunidad; ii) que participa, pues suele ser miembro activo de movimientos cívicos y organizaciones comunitarias; iii) que, con sentido crítico, evalúa permanentemente las estructuras sociales, políticas y económicas; y iv) que enfrenta los desafíos de injusticia y opina sobre los principales problemas de su entorno societal (Westheimer \& Kahne, 2004).

\section{Nota metodológica}

Para reconstruir las experiencias de movilidad académica de las y los jóvenes estudiantes extranjeros que han cursado parte de sus estudios en la Universidad de Valparaíso, hemos usado los relatos de vida (Bertaux, 1997; Cornejo, Mendoza, \& Rojas, 2008; De Miguel, 1996; Pujadas, 1992). Como técnica de investigación, esta se inscribe en el denominado método biográfico, parte de la perspectiva cualitativa de la investigación en ciencias sociales (Vallés, 1998). Dicha técnica considera cada historia singular como portadora de la historia social que la sustenta y la construye, siendo entonces posible acceder a lo social a partir de lo singular. A su vez, se trata de una técnica privilegiada para acceder a fenómenos sociales que se encarnan en las biografías personales, donde el foco del análisis es más longitudinal, vale decir, donde importa reconstruir trayectorias, experiencias acaecidas y las formas en las que se las significa.

Tal como recomienda la literatura, trabajamos con pocos casos, privilegiando un análisis más profundo. En este caso se trató de una muestra de seis estudiantes daneses de pregrado ${ }^{1}$ que cursaron un semestre académico a través de un programa internacional de intercambio de larga data, entre su universidad de origen (Universidad de Aalborg) y la Universidad de Valparaíso, como parte de su experiencia de movilidad estudiantil internacional, quienes fueron entrevistados en sus países de origen una vez que habían regresado. Como se sabe, los muestreos cualitativos operan con pocos casos, por lo que la calidad de la muestra se evalúa más por la saturación y calidad narrativa de los informantes que por los criterios propios de los muestreos probabilísticos. En este caso, el tipo de muestreo que opera está guiado fundamentalmente por razones pragmáticas, en tanto hemos contado con contrapartes que nos han permitido una mayor eficacia en la localización de los estudiantes. Este tipo de muestreo se conoce como muestreo por criterios prácticos o de accesibilidad (Vallés, 1998). El proceso de reclutamiento de los participantes del estudio se realizó a través de informantes clave.

El dispositivo metodológico se diseñó teniendo en cuenta la obligación de realizar dos encuentros con cada participante a partir de una invitación inicial para conversar acerca de su experiencia. El primer encuentro partió con una consigna abierta que permitió al narrador o narradora estructurar el curso y la trama de su relato. Para el segundo, se envió previamente la transcripción del primer encuentro, de modo que pudieran volver sobre sus testimonios de manera más reflexiva y completar los temas confusos, incompletos o más controvertidos. La pertinencia de entregar a cada participante una copia de la transcripción del primer encuentro descansa en que su revisión suele generar más recuerdos, narrativas y sentidos, así como ahondar en aquellos pasajes que le resulten más importantes (Cornejo et al., 2008).

1 Al arribar a Chile para comenzar su estadía en la Universidad de Valparaíso, los seis estudiantes daneses que conformaban la muestra se encontraban cursando estudios de pregrado (Bachelor) en Ciencias Políticas y Economía Internacional en el Departamento de Estudios Globales de su universidad de origen. 
Los relatos fueron analizados mediante el análisis de contenido narrativo. Ello supuso que la producción de las entrevistas requirió su grabación y transcripción íntegras, de forma que cada relato fuera analizado como un caso, para proceder luego a la comparación entre relatos. Para facilitar el análisis triangulado por los diferentes miembros del equipo de investigación, así como la comparación entre casos, los relatos fueron codificados con el programa Atlas.ti.

\section{Resultados}

Para facilitar la comprensión de los resultados los presentamos en dos partes. En la primera se expone la reconstrucción de la experiencia de intercambio en un continuo que va desde una significación múltiple a una más unívoca; luego, se describen las dimensiones de la ciudadanía global que van desde un grado básico de compromiso con el nuevo entorno al que se llega a estudiar hasta un alto grado de involucramiento. La segunda parte se concentra en analizar cómo las y los estudiantes extranjeros construyen el sentido de alteridad tras su experiencia de intercambio, tanto en la forma en que conciben a sus sociedades de origen como a las del destino de la movilidad; para graficarlo, usamos tres metáforas tomadas del imaginario sobre los viajes de los antropólogos en sus acercamientos al «otro» no europeo.

\section{1 De la experiencia a la ciudadanía crítica}

\section{La experiencia múltiple o integral v/s la experiencia singular}

En general los relatos de los y las jóvenes sobre lo que significó la movilidad internacional dan cuenta de una experiencia que rebasa con creces el propósito académico que formalmente se asocia al intercambio estudiantil. Si bien las motivaciones para viajar a Chile varían (académicas, biográficas o incluso circunstanciales), ellos y ellas valoran el haber logrado mucho más que calificaciones o conocimientos. Una de las ideas que emerge con más fuerza es la de haber aprendido «otras cosas importantes» (Lena): desde nuevas percepciones sobre la política a una visión más compleja de las sociedades, pasando por aspectos relacionados con el desarrollo personal y las relaciones familiares. Ejemplo de ello es la descripción que Lena hace de Valparaíso como una ciudad bohemia que le permitió «ensanchar su percepción». A su vez, señala que el viaje motivó un cambio en la elección de sus estudios de posgrado y redefinió su «vocación» de ser periodista (Lena).

«Empecé a pensar diferente del mundo y de la política también porque, en comparación a Dinamarca es muy diferente (...) pero también he cambiado mucho mi percepción de la familia al estar en Chile.» (Lena)

Marco destaca sus impresiones sobre la ciudad de Valparaíso, la cultura chilena y las movilizaciones estudiantiles del 2011, mucho más que las actividades académicas. Su relato comienza con un hecho biográfico ineludible: su madre es chilena, por lo que pensó su intercambio como una oportunidad para profundizar sus conocimientos sobre el país materno. Tras ahondar en su experiencia con el movimiento estudiantil chileno, argumenta cómo reorientó sus elecciones académicas tomando cursos que le permitieran implementar cambios en el país europeo. De regreso en Dinamarca, adquiere compromisos con su comunidad y participa frecuentemente en actividades políticas, algo absolutamente nuevo para él. Asimismo, se compromete con el mundo desde el «pensar global, actuar local» (p. 8). Para él, el movimiento estudiantil fue una expresión de los problemas locales de Chile, pero se expresa simultáneamente a nivel mundial, lo que le induce a aumentar notablemente su compromiso.

El relato de Sarah se organiza en una intriga cuyo centro es su sorpresa ante la desigualdad social que observó en Valparaíso, en contraste con lo que había escuchado decir antes en su país de origen sobre Chile: el «milagro neoliberal». A partir de ello, observa cómo los chilenos abordan su pasado reciente asociado a la dictadura militar (1973-1990), así como el más remoto vinculado al 
período colonial. A diferencia de otros compañeros, no se limita a relatar su experiencia sino que la enmarca en ideas más amplias sobre la sociedad o la cultura. Para Sarah, el viaje a Valparaíso es significativo también desde un punto de vista biográfico-familiar por el compromiso de sus padres con Latinoamérica.

«Una vez nos visitó una mujer de Guatemala que nos contó su historia de cómo habían secuestrado a su marido y el camino que ella hizo para encontrar su marido y ya estaba muerto y esas cosas, esa historia hizo un gran... impacto en mí cuando era pequeña, y hay más cosas similares.» (Sarah)

Vinculada toda su infancia con organizaciones de solidaridad internacional, Sarah no experimenta grandes «shocks» culturales, pero sí romantiza su relación con Latinoamérica al hacerse cargo de los ideales de sus padres. Frente a ello, el viaje a Chile le posibilita una mayor autonomía respecto del legado familiar.

\section{Entre una ciudadanía consciente y una ciudadanía crítica}

Como señalamos antes, en el contexto de un mundo globalizado es posible comprender la ciudadanía en un continuo entre una ciudadanía consciente y una más crítica que va desde un i) actuar responsable en su comunidad; (ii) una actitud participativa en agrupaciones cívicas y/o comunitarias y iii) la conformación de un sentido crítico; hasta iv) el enfrentamiento de las injusticias identificadas (Tarrant, 2011). Entre los entrevistados/as encontramos ejemplos de las 4 formas mencionadas, agrupables más bien en dos grandes categorías a) un actuar responsable y participativo; y b) la evaluación permanente de los problemas de su entorno social.

-Actúa responsablemente en su comunidad y/o participa de movimientos cívicos y organizaciones comunitarias:

Un ejemplo de esta ubicación es el caso de Sisse, quien, una vez en Chile, se concentra en lo académico y no se integra mucho en actividades vinculadas a los problemas sociopolíticos de su entorno. A Sisse los estudios en Chile le resultan más difíciles de lo esperado por la dificultad idiomática, pero también porque se enfrenta a un estilo de docencia que le demanda una gran carga de lectura. Con todo, valora lo mucho que logró aprender. De vuelta en Dinamarca comienza a participar con mayor frecuencia de debates político/sociales a través de Facebook, se afilia a la Cruz Roja y comienza a donar dinero a diversas ONG:

«(...) estoy militando en una organización, yo soy parte de una organización que se llama Cruz Roja, Cruz Roja para niños que tienen menos recursos. Y bueno, sí, yo estoy miembro de diferentes cosas sociales, contribuyo con un poco de dinero, y contribuyo con mis manos por ejemplo he ayudado a hacer comida con niños (...) y, bueno sí, leo mucho más en internet, en periódicos sobre asuntos políticos, sí.» (Sisse)

Lena, por su parte, muestra su apoyo al movimiento estudiantil, a pesar de no ser realmente «su lucha» (Lena, p. 3), lo que fundamenta en el hecho de ser estudiante de sociología. Si bien opina sobre los principales problemas de su entorno social, no parece vivirlos como algo propio, sino como parte de la «lucha» de los estudiantes chilenos. Así narra su participación en las marchas:

«Recuerdo una vez que subieron a Facebook una foto nuestra en la marcha, y una persona comentó algo sobre los "turistas perdidos en la marcha". Eso me hizo re-pensar nuestra participación por un minuto, pero concluí que el comentario era en realidad bastante discriminador, y continuamos participando. Sabíamos que probablemente no era nuestra lucha, pero queríamos mostrar nuestro apoyo en lugar de sólo observar como turistas normales, ya que éramos estudiantes de sociología.» (Lena)

Pero, el cuestionamiento en torno a si el movimiento estudiantil chileno es o no una lucha propia, no es transversal; encontramos también casos de participantes en las marchas que se sienten convocados por otro motivo, como el caso de Morten (19 años): 
«Hasta cierto punto, sentí que, debido a mí situación estudiantil en Dinamarca, no podía permitirme no participar y empeñarme en el proceso o, por lo menos, intentar de comprender el programa, formulado por los estudiantes y las demandas principales.» (Morten)

La participación de Morten en las protestas estudiantiles parecen marcadas por cierto espíritu etnográfico bien intencionado, pero carente de un sentido más crítico, lo que a su vez da pistas sobre la eventual relación entre una actitud responsable y una aproximación al otro un tanto exótica:

«Al estar en Chile por un rato y al entender y aprender de las condiciones bajo las cuales viven estudiantes de otras partes del mundo, uno va a entender que, como estudiante danés, uno tiene el derecho y acceso a bienes que, en varios otros países, parecen utópicos.» (Morten)

El ejemplo de Morten ilustra cómo la experiencia de intercambio permite a los estudiantes encontrar su espacio en una cierta «ciudadanía global», participando de las «luchas ajenas», valorando también sus privilegios como ciudadanos de países desarrollados, lo que induce a pensar si acaso la noción misma de «ciudadano del mundo» no está circunscrita a ciertas condiciones de mayor o menor privilegio.

-Evalúa permanentemente los principales problemas de su entorno social:

En el otro lado, el análisis de la experiencia de intercambio de Sarah, Marco y María contiene un re-pensar la sociedad danesa a partir de lo vivido en Chile. En el caso de Sarah, su indignación ante las injusticias que observa en Latinoamérica le lleva a cuestionar su propio país, y, a diferencia de sus compañeros, no traslada esta impresión al campo profesional o disciplinar, sino que lo aborda desde un sentido ciudadano. Para ella el viaje no involucra un giro en su carrera, sino una experiencia personal que contribuye a su formación política y refuerza sus convicciones:

«Sí, reforzó las cosas que me habían contado mis papás, ya en Chile lo vi cada día en la calle, lo viví, y eso...es más fuerte la experiencia. Y me hizo sentir más solidaridad con Chile, con la gente allá. En Dinamarca los políticos se pelean entre sí, nadie tiene visiones muy diferentes de los demás. Son todos así más o menos similares (entrevistador: ¿Ideológicamente no hay muchas diferencias?) No sí, lo hay, pero en realidad es más... real politics... que ideología. Allá en Chile vi unos movimiento llenos de ideología, luchando para un futuro más igualitario.» (Sarah)

Sarah proviene de una familia que le ha inculcado el valor de la solidaridad, y, aunque no menciona que sus padres militen en partido alguno, construye sobre este valor su posición política beligerante frente a la xenofobia, al neo-liberalismo y a la desigualdad de género, expresando una apertura a lo global que le permite incorporar aspectos de otras culturas.

Marco representa un tipo de ciudadanía en la que se mezcla la crítica y la participación. A partir de su viaje construye un sentido sociopolítico global más crítico que antes de dicha experiencia, especialmente por su implicación con el movimiento estudiantil chileno. Su relato se divide entre un antes y un después de la «la toma» ${ }^{2}$ de la Facultad, durante las movilizaciones estudiantiles del 2011. A partir de este suceso comienza a alejarse de sus compañeros de la facultad y empieza a establecer lazos más fuertes con personas en otros espacios vinculados a diferentes formas de militancia política, lo que contrasta con su realidad en Dinamarca. Sin embargo, de regreso a su país de origen, su estado de ánimo no refleja el alivio de vivir en un país con mayores derechos sociales, sino la necesidad de unirse a un movimiento político; de tomar «acciones concretas»:

«(...) siempre he sentido que la democracia es súper importante, pero yo creo que se ha concretizado más (...); yo y mis amigos, mi familia, los estudiantes de aquí, los estudiantes en Chile, se ha vuelto mucho más concreto y mucho más cerca, de cierta manera ¿no? Y yo creo que el proceso de estar ahí me ayudó a ponerlo en otro marco.» (Marco)

2 El movimiento estudiantil del año 2011 adoptó la estrategia de toma. Se trata de encerrarse en el recinto educacional y utilizarlo como punto de reunión, asamblea u otras actividades asociadas a un paro estudiantil. 
Marco reconoce que no solo participa a través de la militancia política en Dinamarca, sino que intenta transmitir algunas prácticas aprendidas en el movimiento estudiantil chileno, como exhibir documentales, invitar a profesores a hablar sobre cuestiones políticas o cocinar comida chilena.

Finalmente, María, quien se presenta como «hija de trabajadores», reconoce que, al contrario de ella, sus padres nunca tuvieron la oportunidad de viajar y que por ello se siente una privilegiada. Expresa siempre un tono de responsabilidad que la muestra como una joven auto-consciente y reflexiva:

«(...) ellos tenían alrededor de veinte años y yo veinticinco, y sentí que estaba en un lugar en mi vida distinto al que estaban ellos. También creo que ellos me vieron diferente, les pareció que yo era muy vieja cuando dije que tenía veinticinco (...) Para mí, viajar es parte central de mi vida, como también expandir mis horizontes, pero tengo prioridades distintas ahora.» (María)

En general, la épica del relato de María aparece en el conflicto en la formación de su subjetividad política; un construirse a sí misma como sujeto político, más allá de militar en un partido:

«Creo que [ahora] me volví más conservadora, porque, no sé, vengo de una familia de clase trabajadora, son socialistas y creo que eso conlleva una mayor apertura frente al mundo. Pero cuando volví a casa también sentí la urgencia de conservar algunas culturas como eran (...) quería conservar la cultura indígena guatemalteca, la cultura maya (...). No he cambiado, de hecho, pero tengo muchas más perspectivas, ya no sólo mi pequeña caja cuadrada socialista.» (María)

\subsection{Los imaginarios sobre las sociedades de origen y destino y la cuestión de la alteridad}

A partir de lo anterior, presentamos tres miradas sobre «el otro» con el que se convive en un escenario global. Para graficarlo, trabajamos con ciertas metáforas que pretenden problematizar la cuestión de la alteridad: la representación del lugar de destino como un escenario exótico donde los estudiantes hacen las veces de etnógrafos clásicos o aventureros; la observación participante de antropólogos en acción, y la figura del etnógrafo postcolonial, que supone una auto-reflexión, que tiene como centro el país de origen reconsiderado ya no como un espacio natural o neutro sino igualmente marcado por condiciones geopolíticas e históricas.

\section{Los etnógrafos clásicos.}

Tras la experiencia de movilidad, algunos jóvenes interpretan su viaje como una forma de aprender sociología en la práctica (Lena, p. 4), o como un ejercicio de intercambio cultural (Sisse, p. 6), en el que ambos lugares - de origen y destino - están bien delimitados. En tales casos todo es una buena oportunidad para reflexionar, comparar realidades y «ensanchar la percepción» (Lena, pp. 9-10). Así la otredad se asimila desde la curiosidad, intentando mantener una mente abierta, aun cuando ellos y ellas han sido objeto de prejuicios (positivos y negativos) por su extranjeridad asociada al prototipo escandinavo (blanco, rubio, alto). Así, Lena señala que los estudiantes chilenos se mostraban en general amables con ella o sus compañeros de intercambio, pero su experiencia en el espacio público no le permitiría decir que Chile sea un país seguro como suele promocionarse. En este caso la marca de género no es menor.

«bueno si alguien me pregunta así voy a decir que no, pero hay una diferencia también entre hombres y mujeres, porque yo fui con Marco también y cuando estábamos con él nada pasó, pero si íbamos la Carolina y yo, que es rubia también, si estábamos solas, nos miraban así, obvio porque somos diferentes. Creo que hay una diferencia grande y no siempre positiva.» (Lena)

Los estudiantes que ubicamos bajo esta categoría coinciden en ver a su sociedad de origen (Dinamarca) como un lugar para la teoría y Valparaíso (Chile o Latinoamérica) como el lugar de la 
práctica o a lo sumo como la teoría puesta en práctica. En estos casos cobra sentido la metáfora del etnógrafo clásico que sale de su cultura a explorar lo ajeno.

«Chile está en medio de cambios sociales, lo cual significó que las marchas y la toma de la Universidad afectó mucho nuestra estadía y al final no era mucho que estudiábamos en el sentido tradicional. No obstante, aprendí un montón sobre los procesos democráticos, organización, movimientos sociales, historia y en general como funciona la sociedad chilena. De cierto modo, éramos antropólogos en acción y tuvimos la suerte de estudiar por adentro un movimiento estudiantil en acción.» (Marco)

«Como la Universidad estuvo en paro, asistimos a las marchas, lo que fue una experiencia muy interesante. Se hizo claro que, en Chile, aquello que aprendes en sociología puede ser visto físicamente en sociedad. Esto hizo de la experiencia algo mucho más educativo, tanto en el nivel académico como personal.» (Lena)

Los testimonios parecieran reproducir la lógica tradicional que sitúa a Latinoamérica como el lugar que provee datos y a Europa como el espacio para teorizar, postura criticada ampliamente por las posiciones postcoloniales. Otro ejemplo es Morten, quien no tenía un interés previo por Valparaíso, simplemente quería un destino «seguro» a la vez que distinto a los países en los que ya había estado antes (Centro América). Es el único que al comparar el momento en que llegó y el de su partida se detiene en cuestiones como el clima. Junto con eso describe a Valparaíso como una ciudad «relativamente pobre, sucia, etc.» (Morten). Todo su discurso se sostiene en una posición de extranjería. A diferencia de otros, no intenta encontrar su lugar en Chile ni en el movimiento estudiantil, no siente un vínculo en particular con Latinoamérica, y más bien viene «a ver qué pasa» (p. 2). De ahí que la principal motivación de su viaje sea probarse a sí mismo, ver si puede vivir lejos de lo que conoce por medio año.

La mirada cruzada: los «antropólogos en acción».

Esta posición se distingue de la anterior porque los jóvenes sintieron ser agentes, al menos por un tiempo, en su lugar de destino. Una buena ilustración de esta forma de encarnar la relación con la alteridad la representa Marco, quien explícitamente se describe a sí mismo como «antropólogo en acción»:

«Porque estábamos justo en todas estas marchas, en todas estas asambleas con los estudiantes en Chile, con todas estas cosas que estaban pasando, al mismo tiempo estábamos estudiando antropología. Entonces yo podía verme como antropólogo ahí, porque de eso se trata ¿no? De estar en el campo y ver, entender lo que está pasando.» (Marco)

Este interrogarse aparece también cuando se siente responsable por los otros daneses en términos de brindarles una mayor seguridad en la ciudad; así como cuando al entrar en una discoteca sufre una discriminación por ser moreno, al contrario de sus compañeros daneses rubios. Esto lo impulsa a hablar desde una posición de ciudadano global, que se hace más consiente después del viaje y a tomar conciencia de su identidad compleja:

«(...) en Chile soy danés y en Dinamarca soy... o sea ese es un problema de ser ciudadano global ¿no? porque aquí soy extranjero todavía aunque soy danés... porque la gente te mira solamente por la apariencia física.» (Marco)

La narración de Marco se enfoca en los daneses. Su experiencia del viaje, marcada por las movilizaciones estudiantiles del 2011, tiene un fuerte carácter político, y es esa politización la que quiere transmitir a los daneses:

«Yo creo que los daneses son un poco...tienen un poco vergüenza en lo público y son malos para tomar contacto con gente extraña, y los chilenos no tanto, entonces yo creo que es esos valores, no es que no lo tuve antes, es que me di cuenta que los daneses son muy distantes.» (Marco) 


\section{La mirada crítica hacia el país de origen}

Desde esta tercera posición las reflexiones posteriores al viaje a Chile tienen un dejo de reproche a la sociedad de origen. Es el caso de Sarah y María, para quienes, en lo político, Chile en general confirma las impresiones previas que tenían sobre los peligros del neoliberalismo que ya identificaban en su propia sociedad. Sarah organiza su relato reconstruyendo lo que buscaba al iniciar su intercambio. Desde esa lógica, reflexiona sobre la incidencia que tuvo la experiencia de movilidad en sus compromisos políticos, señalando que no cambió su forma de pensar, pero sí le ayudó a fundamentarla mejor (p. 4). En ese sentido, Sarah no se ve superada por la experiencia «intercultural». El viaje a Latinoamérica era para ella un momento lógico en su vida y evalúa haberlo hecho en el momento adecuado (p. 2) como una forma de conocerse a sí misma. No se trata de una «exploradora» maravillada, sino de alguien que aprovecha la instancia para iniciar una búsqueda más interna, que se combina con una mayor reflexividad política:

«Mm, no, no cambió mucho, pero mis argumentos sí cambiaron, porque ahora sabía cómo es la realidad en Chile y es muy diferente de lo que dicen los daneses o los europeos en general. Porque todo el mundo dice que Chile es el modelo para seguir, el «milagro del neoliberalismo» (...) Después de vivir en ese país y ver cómo es, sabía que los números no reflejan la realidad. En mis estudios en Dinamarca esa experiencia fue muy importante porque mis profesores hablan de los números, las cifras, y entonces creen que en realidad todo está bien, que el estándar de vida es muy elevado, y no lo es.» (Sarah)

Sarah sugiere que Latinoamérica es un lugar de acción, un lugar donde «las cosas pasan» (p. 13), pero a diferencia de Marco y Lena, no lo entiende como una oportunidad de poner en práctica sus conocimientos, sino para comprender el contexto y mostrarlo a los daneses. Sarah valora el encuentro con la realidad chilena y con los debates políticos en los que participó como un aporte a su autoconstrucción como sujeto y al fortalecimiento de sus argumentos acerca de lo que quiere preservar y lo que quiere cambiar en el mundo:

«Lo que me gusta de mi cultura es que los hombres y las mujeres son iguales, tenemos casi los mismos derechos (...). Es algo que querría cambiar en Latinoamérica, porque como yo lo veo las mujeres son poco valoradas (...) y hay mucha violencia doméstica.» (Sarah)

A modo de contraste quizá con la posición del etnógrafo clásico, Sarah es la única que alude al eurocentrismo como una amenaza a sus ideas, vale decir, aunque ella es sensible a las desigualdades y violencias de género que afectan a las mujeres en su lugar de destino, no por ello quiere replicar los modos en que los europeos de antaño han pretendido mostrar a las poblaciones de Latinoamérica los problemas que les afectan y los mejores modos de solucionarlos. Sarah muestra cómo en ella están operando los conocidos debates entre universalismo y multiculturalismo a la hora de pensar los derechos.

«trato de mantener el equilibrio y no viajar para decir a otras personas como tienen que vivir, eso no es lo que quiero, porque así han sido los europeos durante décadas, siglos.» (Sarah)

Finalmente, aunque en el relato de María aparecen varios temas ya revisados, lo que destaca en ella — como en Sarah - es su convicción de haber comprobado que el neoliberalismo es un peligro para Dinamarca. María es mayor que el resto de los estudiantes y su viaje a Chile es el último de los que ha hecho a Latinoamérica. Es la única que expresa que con su viaje se decepcionó de Dinamarca, y hace de este tema un tópico importante en la entrevista:

«No quiero que en Dinamarca las cosas sean como en Chile, y Chile es un ejemplo, ¿no? Porque todo está privatizado; los hospitales, las escuelas, la educación en general... necesitas dinero para hacer cualquier cosa en Chile, y creo que la gente aquí [Dinamarca] no se da cuenta de que si no somos cuidadosos, si no luchan, si no hacen algo, si no se alzan y dicen algo y marchan por las calles como lo hacen en Chile, aquí va a ser igual.» (María) 


\section{Reflexiones finales}

La literatura sobre los procesos de migración económica ha problematizado cómo las personas que emprenden nuevos rumbos se ven enfrentadas a procesos de reinterpretación y ajuste de su ciudadanía, entre otras razones porque entre los lugares de origen y destino se evidencia diferentes y desiguales formas de ejercer, interpretar y disputar los derechos. Sin embargo, no solemos pensar que esto también ocurre con las y los jóvenes que cursan parte de sus estudios en el extranjero, ni cómo esa experiencia puede contribuir a sentidos globales de la ciudadanía. Muy al contrario, cuando pensamos en la movilidad estudiantil universitaria solemos reducirla a sus beneficios académicos, $y$, especialmente, a la ventaja que supone vivir un tiempo en el extranjero para acumular nuevas experiencias. Como hemos mostrado en este trabajo, la incorporación de la dimensión internacional a las tareas de la educación universitaria aporta no sólo en la diversificación de las trayectorias académicas y personales de los y las estudiantes, sino que también altera sus dimensiones ciudadanas; esto es, los referentes de sentido con que se evalúa lo relativo a la vida en común.

Efectivamente, en este trabajo hemos podido avanzar en comprender cómo el estudiar en el extranjero contribuye a transformar los valores, las normas y los comportamientos sociopolíticos. En particular, nos concentramos en los debates sobre la ciudadanía global. Específicamente en cómo, desde ese lente, podíamos interpretar en los relatos de vida de estudiantes daneses que cursaron durante el año 2011 un semestre en la Universidad de Valparaíso el impacto de esta experiencia en las trayectorias de vida de jóvenes de procedencia nórdica y cultura socialdemócrata, cuando se enfrentan a un contexto como el chileno, que en los últimos años ha sido remecido por las movilizaciones estudiantiles que reclaman la educación como un derecho. Con ello pudimos constatar cómo operaban, en este caso específico, ciertas ideas clave que aparecen en la literatura.

Lo primero que los relatos muestran — tal como indica Van Mol— es que los programas de estudio en el extranjero suponen una experiencia que rebasa los límites de la formación académica. Como mostramos, solo uno de los casos reduce a ese ámbito lo significativo del viaje. Pudimos constatar también que estudiar en el extranjero promueve una conciencia de los problemas mundiales, como ha argumentado Dolby, en este caso, en lo referido a los impactos de los procesos de neoliberalización en el acceso y calidad de la educación universitaria, entendida como derecho universal, algo que los y las jóvenes problematizan al contrastar la situación de la educación en su país de origen con lo que sucede en el país que los acoge.

A través de los relatos también pudimos ilustrar cómo funciona aquello que varios autores resaltan sobre el potencial que supone este tipo de movilidad en la confrontación de barreras y prejuicios etnocéntricos, en tanto los y las estudiantes transitan desde un entorno familiar a uno imprevisto, lo que efectivamente conduce a una conciencia diferente de sí mismo y de los demás, pero en un proceso que no es lineal sino multidimensional. En los relatos esto queda de manifiesto en temas como la desigualdad de género o en la vivencia personal de prejuicios asociados a procesos de racialización que las y los jóvenes experimentaron en carne propia al vivir en Valparaíso.

Pero, además la estadía en Chile tuvo un componente adicional: a todos los y las jóvenes les correspondió vivir un proceso de alta movilización estudiantil, marcado por marchas, protestas y tomas de edificios universitarios asociados a las demandas estudiantiles por una educación pública, gratuita, de calidad y no sexista. Tratándose de jóvenes que se insertaron en una carrera de sociología, este hecho provocó en todos una evidente politización. Es ahí donde podemos concluir, a modo de hipótesis teórica, el interés que puede tener para futuras investigaciones considerar el aporte de Tarrant sobre la ciudadanía global. Para ello, proponemos una figura (Figura 1) que se arma con el cruce de dos ejes que ahora pasamos a describir. 
Figura 1. Las cuatro dimensiones de la ciudadanía global

\section{Ciudadanía Crítica}

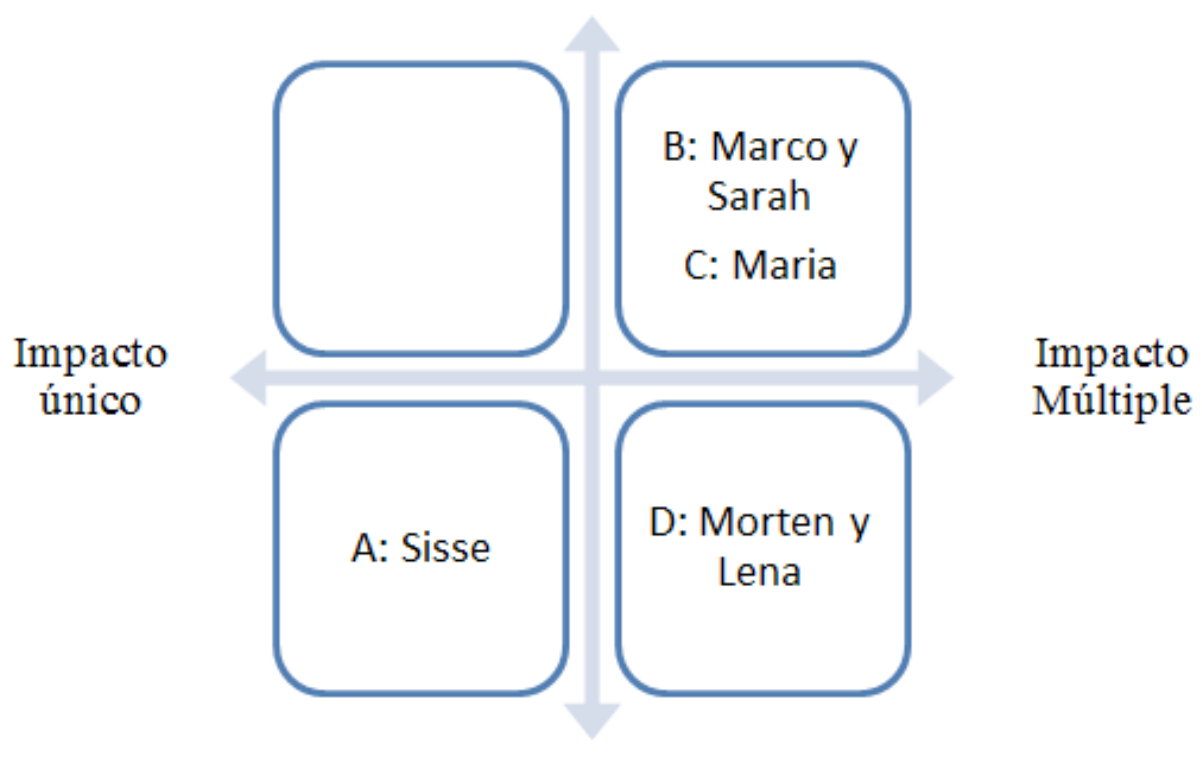

\section{Ciudadanía Responsable}

Nota. Elaboración a partir de la clasificación de Tarrant (2011).

Como podemos observar en el gráfico, la experiencia de intercambio se reconstruye como una vivencia que aporta a cada joven diferentes orientaciones de sentido personal, académico y político. En los resultados que presentamos, solo en un caso la movilidad se significa como un logro meramente académico (eje 1 del gráfico); todos los demás se ubican en lo que entendemos como el impacto múltiple, de forma que las cuatro maneras de experimentar la ciudadanía global no se presentan de manera gradual y acumulativa, como parece sugerir Tarrant, sino como dimensiones claramente interrelacionadas.

El segundo eje permite entender la ciudadanía global moviéndose entre dos polos. En uno ubicamos las dimensiones relacionadas con los atributos 1 y 2 del modelo de Tarrant (referidas a los valores de una ciudadanía responsable) y la metáfora del ciudadano («buen samaritano») mientras que en el otro extremo se las características 3 y 4 (que indica que se enfrenta críticamente los problema estructurales de las sociedades de origen o enfrenta los desafíos de la injusticia como parte de un entorno que incluye a Europa y Latinoamérica) que condensamos en la figura de un ciudadano global más crítico.

Al interior de cada cuadrante ubicamos a quienes entrevistamos siguiendo la lógica de cómo operan los imaginarios sobre las sociedades de origen y destino, y la cuestión de la alteridad que ya describimos bajo las distintas metáforas etnográficas. Con ello podemos concluir que —al menos en unos casos - la experiencia de intercambio no desactiva la visión eurocéntrica, sino que más bien la renueva; se trata ahora de una experiencia de crecimiento personal en un lugar exótico, en contraste con un mundo social que les permite valorar los derechos sociales de su país de origen. Nada de esto supone una mayor implicación con los conflictos que observaron en el lugar de destino o un cuestionamiento a sus sociedades de origen.

En otros casos, por el contrario, la movilidad es una situación que pone en jaque el propio lugar de origen y las relaciones globalizadas sobre las que se articula la desigualdad geopolítica, lo que sugiere que la idea de una ciudadanía responsable - en términos de Tarrant - no está exenta de 
una mirada hacia el «otro» que reproduce ciertas lógicas coloniales. Se trataría de otra forma de ciudadanía más crítica y exigente, que experimenta la relación con el otro desde una posición menos apegada a estereotipos tradicionales. Esto implica, por un lado, que la estadía en países marcados por mayores desigualdades sociales y con escenarios políticos donde surgen movimientos sociales que las cuestionan, puede ser un escenario privilegiado para observar los conflictos políticos y sociales en un mundo globalizado. Por otro lado, como hipótesis a revisar en futuras investigaciones, los casos analizados permiten aventurar, además, que esa ciudadanía más crítica y su relación con la alteridad postcolonial también pareciera estar asociada a trayectorias familiares, donde los padres y madres experimentaron diferentes modos de relación con sociedades tradicionalmente entendidas como subdesarrolladas desde posiciones más o menos políticamente comprometidas con el devenir de tales países.

\section{Lista de referencias}

Bell, K., Moorhead, B., \& Boetto, H. (2015). Social work students' reflections on gender, social justice and human rights during a short-term study programme to India. International Social Work. Recuperado de: https://doi.org/10.1177/0020872814559559

Bertaux, D. (1997). Les récits de vie. Paris: Editions Nathan.

Bowman, K. S., \& Jennings, A. (2005). Pura vida: Using study abroad to engage undergraduate students in comparative politics research. PS: Political Science and Politics, 38(1), 77. Recuperado de: http://www.jstor.org/stable/pdfplus/30044230.pdf

Castel, R. (2006). La inseguridad social. ¿Qué es estar protegido? Buenos Aires: Manantial. http://www.psykhe.cl/index.php/psykhe/article/view/326

Cornejo, M., Mendoza, F., \& Rojas, R. C. (2008). La investigación con relatos de vida: pistas y opciones del diseño metodológico. Psykhe, 17(1), 29-39. Recuperado de http://www.redalyc.org/ articulo.oa? id $=96717104$

De Miguel, J. M. (1996). Auto-biografías. Madrid: Centro de Investigaciones Sociológicas.

Doerr, N. M. (2016). Time and the other politics of cultural others study abroad away and going home eventually. Journal of Cultural Geography, 33(1), 51-61. Recuperado de: https://doi.org/10.108 0/08873631.2015.1065031

Dolby, N. (2007). Reflections on nation: American undergraduates and education abroad. Journal of Studies in International Education, 11(2), 141-156. Recuperado de:

http://journals.sagepub.com/doi/abs/10.1177/1028315306291944

Giddens, A. (1994). Consecuencias de la modernidad. Madrid: Alianza.

Habermas, J. (1986). Problemas de legitimación en el capitalismo tardío. Buenos Aires: Amorrortu.

Hunter, B., White, G. P., \& Godbey, G. C. (2006). What does it mean to be globally competent? Journal of Studies in International education, 10(3), 267-285. Recuperado de: http://journals. sagepub.com/doi/abs/10.1177/1028315306286930

Knight, J. (1999). Issues and trends in internationalization: A comparative perspective. En S. Bond, \& J. P. Lemanson (Eds.), A new world of knowledge: Canadian universities and globalization, (pp. 152-170). Otawa: International Development Research Center.

Lörz, M., Netz, N., \& Quast, H. (2016). Why do students from underprivileged families less often intend to study abroad? Higher Education, 72(2), 153-174. Recuperado de: https://www.altmetric. com/details/4628755 DOI: 10.1007/s10734-015-9943-1

Luchilo, L. (2006). Movilidad de estudiantes universitarios e internacionalización de la educación superior. Revista CTS, 7(3) 105-133. Recuperado de: http:/www.scielo.org.ar/scielo. php?pid=S1850-00132006000100007\&script=sci_arttext\&tlng=pt

Luhmann, N. (1990). World society as a social system. En N. Luhmann(Ed.), Essays on self-reference, (pp. 175-190). New York: Columbia University Press. 
Melucci, A. (2010). Acción colectiva, vida cotidiana y democracia. México, D. F.: El Colegio de México.

Mendoza, C., Staniscia, B., \& Ortiz, A. (2016). Migración y movilidad de las personas calificadas: nuevos enfoques teóricos, territorios y actores, Biblio 3W. Revista Bibliográfica de Geografía y Ciencias Sociales, XXI(1116) [en línea].

Muñoz, L. (2004). El referente de la internacionalización y sus inherencias para la educación superior. Revista de Educación, 29(2), 11-33. Recuperado de: http://www.redalyc.org/html/440/44029202/

Nguyen, A. M. (2012). Study abroad's contribution to critical thinking and world citizenship. Think, 11(31) 27-40. Recuperado de:

https://doi.org/10.1017/S1477175612000097

Pugh, J. D. (2013). The short-term «Bridge model study abroad program: Peacebuilding in Latin America. PS: Political Science \& Politics, 46(4), 791-796. Recuperado de: https://doi. org/10.1017/S1049096513001108

Pujadas, J. J. (1992). El método biográfico: el uso de las historias de vida en ciencias sociales. Madrid: Centro de Investigaciones Sociológicas.

Ritzen, J. M. (2006). Scenarios for higher education, 2020 or when will China invade Iran? Athens: OECD.

Rodríguez, R. (2005). Migración de estudiantes: un aspecto del comercio internacional de servicios en la educación superior. Papeles de Población, (44), 221-238. Recuperado de: http://www. redalyc.org/articulo.oa?id=11204409

https://www.google.com/search?q=Salamanca\%2C+A.+y+Mart\%C3\%ADCrespo\%2C

Servicio de Información de Educación Superior-Sies. (2013). Base de Datos Académicos 2013. Base de Datos Matriculados 2013. Compendio Histórico: evolución de la Matrícula Total por Región (1983-2010). Recuperado de: http://www.sies.cl

Stern, P. C. (2000). Toward a coherent theory of environmentally significant behavior. Journal of Social Issues, 56, 407-424.

Tarabini, A., \& Bonal, X. (2011). Globalización y política educativa: los mecanismos como método de estudio. Revista de Educación, (355), 236-256.

Tarrant, M. A. (2011). A conceptual framework for exploring the role of studies abroad in nurturing global citizenship. Journal of Studies in International Education, 14(5), 433-451. Recuperado de: http:/www.discoverabroad.uga.edu/research/Tarrant\%202010.pdf

Unesco. (2009). Conferencia Mundial sobre la Educación Superior 2009: la nueva dinámica de la educación superior y la investigación para el cambio social y el desarrollo. Recuperado de: http://www.unesco.org/education/WCHE2009/comunicado_es.pdf

Ungar, S. J. (2016). The study-abroad solution. Foreing affairs, (4), 111-124. Recuperado de: https:// www.foreignaffairs.com/articles/united-states/study-abroad-solution

Vallés, M. (1998). Técnicas cualitativas de investigación social. Madrid: Síntesis.

Van Mol, C. (2017). European mobile students, (trans)national social networks, and (inter)national career perspectives. En M. Van Riemsdijk, \& Q. Wang, (Eds.), Rethinking international skilled migration, (pp. 54-74). Oxon: Routledge.

Westheimer, J., \& Kahne, J. (2004). Educating the 'good' citizen: Political choices and pedagogical goals. Recuperado de: www.apsanet.org 